\title{
Novel nanopowder spray shows promising results in humans for the treatment of acute peptic ulcer bleeding
}

The first human study of a novel nanopowder spray to achieve hemostasis was recently published, reporting promising results with an efficacy of $95 \%$ in patients with acute peptic ulcer bleeding.

"Current endoscopic therapies have their limits", says Joseph Sung, lead author. "They are skill-demanding, provide temporary hemostasis and are difficult to apply to bleeding lesions that cover a wide area." Animal studies of this novel nanopowder spray with clotting abilities have reported encouraging results and this led Sung and colleagues to conduct a proof-of-concept investigation to test its safety and efficacy in humans.

Their prospective, single-arm, pilot study included 20 patients with confirmed active peptic ulcer bleeding. After upper gastrointestinal endoscopy, nanopowder spray was administered to the affected area within $24 \mathrm{~h}$ of hospital admission and once the patient had become hemodynamically stable. The bleeding site was observed periodically to evaluate success of hemostasis.

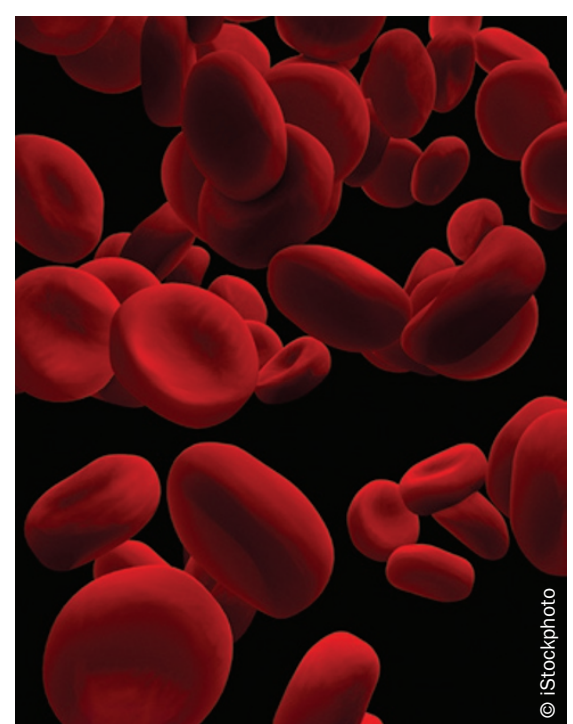

Sung acknowledges the limitations of their study-its non-randomized unblinded nature, small sample size and lack of patients with active spurting bleeding. However, he believes the results are encouraging. "The majority of patients achieved hemostasis and no clinically significant adverse events were reported".

Larger studies to compare the spray with other hemostatic modalities are needed to confirm the efficacy of this treatment. Sung hopes that the value of hemospray will also be assessed for the treatment of other bleeding lesions, for example vascular ecstasia, hemorrhagic gastritis and ulcerative tumors.

Rachel Thompson

Original article Sung, J. J.Y. et al. Early clinical experience of the safety and effectiveness of Hemospray in achieving hemostasis in patients with acute peptic ulcer bleeding. Endoscopy 43, 291-295 (2011) 